\title{
Pulsar Timing Arrays
}

\author{
K. Lazaridis ${ }^{1,2 *}$ \\ ${ }^{1}$ Max-Planck-Institut für Radioastronomie Auf dem Hügel 69, 53121, Bonn, Germany \\ ${ }^{2}$ Department of Physics, Section of Astrophysics, Astronomy and Mechanics, Aristotle University, \\ Thessaloniki, Greece
}

E-mail: klazaridegmail.com

\begin{abstract}
Neutron stars are unique stellar remnants with extreme properties. Their study can be the key to a number of unanswered problems in fundamental physics and astronomy, ranging from stellar evolution to strong field gravity. The class of millisecond pulsars have an especially stable rate of pulsation making them some of the most reliable clocks in the universe. Frequent observations of an array of several tens of these objects by the largest radio telescopes on Earth could lead to the first direct detection of gravitational waves. In this paper an overview of the current state, efforts and future prospects of the pulsar timing array consortia will be given. In addition some of the scientific results from the European Pulsar Timing Array (EPTA) collaboration will be presented.
\end{abstract}

From Antikythera to the Square Kilometre Array: Lessons from the Ancients,

12-15 June 2012

Kerastari, Greece

${ }^{*}$ Speaker. 


\section{Introduction}

Pulsars are highly magnetised (dipolar magnetic field $\sim 10^{12} \mathrm{G}$ ) fast rotating neutron stars (NS) with a surface temperature of $10^{6}-10^{7} \mathrm{~K}$, emitting radio pulses along the magnetic axis. If Earth lies in the path of the beam of this "cosmic lighthouse" we can observe its pulses arriving on each rotation, reflecting its spin period. Today, we believe that a typical neutron star has a radius of $10-20 \mathrm{~km}$, a mass of $1.4 \mathrm{M}_{\odot}$ and mean density of $6.7 \times 10^{14} \mathrm{~g} \mathrm{~cm}^{-3}$. It consists of a neutron fluid in equilibrium with $\sim 5 \%$ protons and electrons and an outer solid crystalline lattice (crust) of about $1 \mathrm{~km}$ thick, made of heavy nuclei on the surface and neutron rich heavy nuclei below (see e.g. [1]). The period of most pulsars is around one second while their intrinsic periodicity is extremely stable with period derivatives of $\sim 10^{-15} \mathrm{ss}^{-1}$ (see e.g. [2]).

Among the known populations of pulsars there is the distinct one of millisecond pulsars (MSPs). These are normal pulsars which have been recycled by mass accretion from a stellar companion while the latter was in the red giant phase. During the accretion the loss of orbital angular momentum of the system is converted into an angular momentum gain for the pulsar which spins-up. During this long timescale process the pulsar is becoming a millisecond pulsar and tidal forces circularise the orbit. The envelope of the companion is exhausted and it usually stabilises to a low-mass helium white dwarf (HWD) ([3] and references therein). The period derivatives of millisecond pulsars are observed to be $\sim 10^{-20} \mathrm{ss}^{-1}$ (see e.g. [2]) which makes them extremely stable cosmic clocks. In addition, compared to normal pulsars they do not show increased intrinsic instability (timing noise), which affects the timing precision [4]. These are the main reasons that MSPs are the optimal targets for high precision pulsar timing observations.

High precision timing is the regular monitoring of the rotation of a neutron star by tracking the times of arrival (TOAs) of the radio pulses. The general procedure of pulsar timing (see e.g. [2]), demands a radio telescope to observe the source, a hardware or software processing of the signal in order to de-disperse it and a high signal-to-noise ( $\mathrm{S} / \mathrm{N}$ ) (or a synthetic Gaussian) template profile to cross-correlate the observed pulsar profiles with and get the TOAs. The differences between the observed and expected TOAs are called timing residuals. Finally you can fit the residuals with functions representing errors in the model spin, astrometric and binary parameters.

The pulsar timing technique has been used successfully to deduce the properties of NS and of their binary systems. One use is to do astrometry of MSPs such as PSR J1012+5307 where parallax and 3D velocities were obtained in order to track the evolutionary path of the pulsar on the Galactic plane [5]. However, the most important results have been obtained towards the tests of general relativity (GR) and alternative theories of gravity in the strong field regime with the use of pulsar timing of MSPs in NS-NS or MSP-WD systems. The most stringent test of GR in the strong field regime was provided by observations of the double pulsar system PSR J0737-3039A/B, where both pulsars could be observed in radio (currently one of the pulsars is not detectable) [6], [7].

\section{Detecting Gravitational Waves}

One of the main predictions of Einstein's general theory of relativity is the existence of gravitational waves (GWs). Gravitational waves are ripples in the curvature of space-time generated from the accelerated masses in it, propagating as waves. Generally, gravitational waves are radiated over 
a wide range of frequencies by accelerated objects, provided that the motion is not perfectly symmetric. Although, strong indirect evidence of their existence has been obtained by binary pulsar experiments (i.e. by measuring the orbital shrinkage of $1 \mathrm{~cm} /$ day of PSR B1913+16 [8]), a direct detection has not been yet achieved.

One approach of detecting gravitational waves are the ground based interferometers such as the current LIGO [9] and the future Advanced LIGO [10] and space mission LISA [11]. These are gravitational wave detectors sensitive to the high frequency regime of the gravitational wave spectrum (i.e. Advanced LIGO $\sim 100 \mathrm{~Hz}$, LISA $\sim \mathrm{mHz}$ ), i.e. from coalescing stellar-mass binaries.

An alternative, but complementary to the previous, technique to observe gravitational waves is with a pulsar timing array (PTA). Let us assume that the solar system barycentre and a distant pulsar are opposite ends of an imaginary arm in space. Then the pulsar can be used as a reference clock sending regular signals observed on Earth. If a gravitational wave passes, it will perturb the local space-time metric and the observed effect will be a change in the apparent rotational period of the pulsar. Such a detector will be sensitive in the nano-Hertz regime since the limiting frequency corresponds to the time span of the data sets used ( $\sim 1-10$ years.). If this arrangement is extended with the use of high precision timing data from more millisecond pulsars (more stable clocks) distributed across as much of the sky as possible [12], we have a PTA. With a PTA we have the capability to cross-correlate the residuals for pairs of pulsars $[13,14]$ in order to distinguish the noise in the timing data from the signature of the stochastic gravitational wave background (GWB). The latter, has been predicted to originate i.e. from coalescent super massive black holes in the early universe, from relic GWs form the big bang and from cosmological sources like cosmic strings, inflation and phase transitions ([15] and references therein).

The expected correlated signal is very weak with the rms of the timing residuals in the nanosecond regime. At the moment only a few MSPs have been timed with such high accuracy. It has been predicted that observations of 20-40 MSPs over a period of 5-10 years with consistent rms of $\sim 100 \mathrm{~ns}$ from the timing residuals will be sufficient to significantly distinguish a GWB signal in the nano-Hertz regime [13].

\section{Pulsar Timing Arrays}

As mentioned above we can reach the needed timing precision of $\sim 100 \mathrm{~ns}$ with only a few MSPs and which makes the direct detection of GWs with a PTA very challenging. In order to increase the timing precision we need large radiotelescopes observing with large bandwidths many strong, short period MSPs for long periods of time. In addition, in order to maintain this timing precision for many years other issues have to be dealt with, such as monitoring of the interstellar medium (ISM) and of local telescope problems i.e. instrument instabilities. The former can be achieved by observing the selected MSPs at several different frequencies and the latter by observing the sources with different radiotelescopes.

In order to keep up with all these challenging tasks three major research groups have been formed. a) The Parkes Pulsar Timing Array (PPTA) in Australia which uses the Parkes $64 \mathrm{~m}$ radio telescope observing $\sim 20$ pulsars with allocated time of 100 hours per month [16]. The PPTA combines observations with theoretical work on fields such as sources of GWs and detection methods. b) The European Pulsar Timing Array (EPTA) network which is a collaboration between the 
five institutes (ASTRON, JBO, INAF, MPIfR and Nançay observatory) operating the five largest radiotelescopes in Europe [17]. It is consisting of the Effelsberg 100m radiotelescope of the MaxPlanck-Institute for Radioastronomy (MPIfR) in Germany, the 76m Lovell radiotelescope of the Manchester University, at Jodrell Bank, UK, the 94m equivalent Westerbork Synthesis Radio Telescope (WSRT) of ASTRON in the Netherlands, the 94m equivalent Nançay decimetric radio telescope (NRT) in France and the $64 \mathrm{~m}$ Sardinia Radio Telescope (SRT) in Italy. The EPTA telescopes are observing 23 MSPs with allocated time ranging from 24-250 hours per month depending on the telescope. Apart from the observational efforts the EPTA is also concentrating on theoretical work on detection methods, sources of GWs, cosmic strings and tests of theories of gravities. These theoretical efforts are being led by the Albert Einstein Institut and the MPIfR in Germany and the University of Birmingham and University of Manchester in UK. c) The North American Nanohertz Observatory for Gravitational Waves (NANOGrav) collaboration in North America, which takes data from $\sim 23$ MSPs with the Arecibo $305 \mathrm{~m}$ and Green Bank $100 \mathrm{~m}$ telescopes with allocated times 8 and 18 hours per month respectively [18]. The NANOGrav collaboration combines observational efforts with theoretical work on several fields such as interstellar medium mitigation and detection methods.

The aforementioned groups have developed a global collaboration the International Pulsar Timing Array (IPTA). The goal is to share knowledge and resources in order to achieve the goal of a direct GW detection [19].

The amplitude of a stochastic GWB originating from coalescent super massive black holes in the early universe is expected to be $\sim 10^{15}$ [13]. Currently best upper $2 \sigma$ limits for the amplitude of a stochastic gravitational wave background signal have been obtained from all the three PTAs. The PPTA using its best 7 pulsars with 2-4 years of data obtained an upper limit of $A<11 \times 10^{-15}$ [21]. The NANOGrav used 17 pulsars with a time span of 5 years to obtain a limit of $A<7 \times 10^{-15}$ [22]. The EPTA used data from 5 pulsars over a time span of 5-8 years to obtain the current best upper limit of the GWB amplitude $A<6 \times 10^{-15}$ [23]. It is clear that these limits have not yet reached the sensitivity needed to achieve a direct detection of GWs.

A boost in sensitivity is expected by the EPTA collaboration with the use of the Large European Array for Pulsars (LEAP). The LEAP project is funded through an European Research Council Advanced Grant to Michael Kramer and it is planning to combine the 5 EPTA telescopes to form a phased array (telescope with equivalent size of a $200 \mathrm{~m}$ dish) with a large sky coverage [20]. This will increase significantly the precision of pulsar timing improving the current GWB upper limit of the EPTA. The observing strategy is to observe 20-30 MSPs over a 24-hour period on a monthly basis at $1.4 \mathrm{GHz}$. Observations combining Effelsberg and Westerbork for 12 hours started in July 2011 and in February 2012 the Lovell telescope was added in 24-hour observations. These test observations already show a significant increase in sensitivity. Currently the Nançay radio telescope is being added and Sardinia will follow soon.

\section{Science from the EPTA}

Apart from the ongoing technical effort in the direct detection of GWs exciting science is being done using the MSP timing data from a PTA. In this section some of the scientific results from the EPTA will be presented. 
As mentioned before, cosmic strings are another potential for detection GW source for PTAs. Determining the tension $\mathrm{G}_{\mu}$ will give us a direct observational result of the energy scale of the phase transition that created them in the early Universe, or even the value of the string coupling and the compactification and warping scales in braneworld scenarios. Using the EPTA limit on the SGWB a robust and conservative constraint on the string tension was set, a rather complicated task due to the large number of unknown parameters that get involved in such investigations. The projected constraint of the LEAP project, if not detecting cosmic strings, will be able to provide the most stringent ever constraints on $G_{\mu}$, surpassing the performance of any other present or future CMB experiment. Details of this work can be found in [24].

We already pointed out that MSPs can be used as ideal laboratories for testing GR in the strong field regime. In addition, MSP-WD systems can be used to set constrains on alternative gravity theories. This was the case with PSR J1738+0333, a $5.85 \mathrm{~ms}$ pulsar in a 8.5 hour orbit with a low-mass white dwarf companion. Following a 10 year high precision timing campaign an orbital period decay $\left(\dot{P}_{b}\right)$ was measured which allowed a stringent limit on the emission rate for dipolar GWs. For such an experiment the timing precision is crucial in order to get a precise measurement of $\dot{P}_{b}$ and in the case of PSR J1738+0333 it was achieved mostly because of the high quality data from the Arecibo radiotelescope. Specifically, PSR J1738+0333 provided the most constraining limit for (conformally-coupled) scalar-tensor theories of gravity. In addition, the most constraining test of Tensor-Vector-Scalar (TeVeS)-like theories when the quadratic matter-scalar coupling constant $\beta_{0} \geq|1|$. Details of this work can be found in [25].

The final example of MSP scientific work concerns the exotic category of black-widow pulsars. Specifically, timing data of 13.5 years were analysed of PSR J2051-0827, a black-widow pulsar in a tight orbit with an unknown companion. It was shown that the variations of the orbital period and projected semi-major axis of the binary system are far more extreme than described in earlier work. After an investigation of the possible causes for these variations it was found that a combination of gravitational quadrupole coupling and spin-orbit coupling are the most likely origins of this behaviour. Details of this work can be found in [26].

\section{Future Prospects}

In this section the future efforts and developments towards the direct detection of GWs from PTAs will be briefly presented.

Currently an Ultra-broad Band receiver (UBB) is being tested at Effelsberg $100 \mathrm{~m}$ radiotelescope, in Bonn. It was designed by Weinreb (JPL/TDP) and the MPIfR and funded by an ERC Consolidated Grant to P. Freire. It is a cryogenically cooled receiver which will provide an amazing frequency coverage from $600-3000 \mathrm{MHz}$, where possibly the $70 \%$ of it will be usable (the losses are caused from RFI mostly at low frequencies). Apart from the improvement in the precision of timing it will be able to do coherent dedispersion of the full band providing a great tool for dealing with the ISM issues.

A key in the detection of GWs is to discover more "good" MSP timers. This will be achieved with the ongoing and future pulsar surveys. The first one is the High Time Resolution Legacy Survey with the Effelsberg in the North and Parkes radiotelescope in the Southern hemisphere. It is a survey using 7 and 13 beam receivers at $1.4 \mathrm{GHz}$ with high time and frequency resolution. It is go- 
ing to probe 8 times more volume of the sky and it is going to be deeper than any previous survey. The expected MSPs to be discovered are $\sim 130$. The second expectation for new MSP discoveries is the LOw Frequency ARray (LOFAR) in Europe. LOFAR is built by the Netherlands astronomical foundation ASTRON and operated by ASTRON's radio observatory and has the main core in Netherlands, 6 stations in Germany and one each in Great Britain, France and Sweden. Apart from the new MSPs expected to be discovered by LOFAR precise measurements of delays due to interstellar dispersion will be made for pulsars. Details for LOFAR can be found in the contribution of P. Lambropoulos (current conference proceedings). Finally, the future Square Kilometre Array (SKA) will be the best tool for discovering new MSPs ( 1200 expected) and timing them. SKA is scheduled to function around 2022 and the detection of GWs with pulsars is one of the two key science projects for Phase 1. More details for SKA can be found in the contribution of R. Schillizi (current conference proceedings).

\section{Summary}

In this paper an introduction on the pulsar timing technique and the challenges and efforts towards a direct detection of GWs with a PTA was given. Specifically, the current status of the 3 PTA collaborations (PPTA, EPTA and NANOGrav) was presented and in addition the international collaboration of those the IPTA. The LEAP telescope array of the EPTA was briefly discussed which will provide equivalent sensitivity with the Arecibo radiotelescope, however with much more sky coverage. Some EPTA scientific results were also briefly presented. This exciting science was produced by analysis of long-term multi-telescope MSP data. Finally some of the most important future projects were presented that will definitely give a boost in the precision of pulsar timing and will increase the number of known MSPs.

\section{Acknowledgments}

I am very grateful to all staff at the Effelsberg, Jodrell Bank, Nançay and Westerbork radio telescopes for their help with observations used in this work. I want to thank all the members of the EPTA, IPTA and LEAP for the work they are doing and for sharing it. Part of this work is based on observations with the $100 \mathrm{~m}$ telescope of the Max-Planck-Institut für Radioastronomie (MPIfR) at Effelsberg. Access to the Lovell telescope is supported through an STFC rolling grant. The Nançay radio telescope is part of the Paris Observatory, associated with the Centre National de la Recherche Scientifique (CNRS), and partially supported by the Région Centre in France. The Sardinia Radio Telescope is a project of the Italian National Institute for Astrophysics (INAF). The Westerbork synthesis radio telescope is operated by the Netherlands Foundation for Research in Astronomy (ASTRON) with support from the NWO. The LEAP project is funded through an European Reseach Council (ERC) grant to M Kramer.

\section{References}

[1] Lyne, A. G. and Graham-Smith, F., Pulsar Astronomy, Cambridge University Press, Cambridge 2006

[2] Lorimer, D. R. and Kramer, M., Handbook of Pulsar Astronomy, Cambridge University Press, Cambridge 2005 
[3] Lorimer, D. R., Binary and Millisecond Pulsars, Living Reviews in Relativity, (2005)

[4] Verbiest, J. P. W., Bailes, M., Coles, W. A., Hobbs, G. B., van Straten, W., Champion, D. J., Jenet, F. A., Manchester, R. N., Bhat, N. D. R., Sarkissian, J. M., Yardley, D., Burke-Spolaor, S., Hotan, A. W., You, X. P., Timing stability of millisecond pulsars and prospects for gravitational-wave detection, MNRAS, 400, 951, (2009)

[5] Lazaridis, K. , Wex, N. , Jessner, A. , Kramer, M. , Stappers, B. W. , Janssen, G. H. , Desvignes, G. , Purver, M. B. , Cognard, I. , Theureau, G. , Lyne, A. G. , Jordan, C. A., Zensus, J. A., Generic tests of the existence of the gravitational dipole radiation and the variation of the gravitational constant, MNRAS, 400, 805, (2009)

[6] Kramer, M. , Stairs, I. H. , Manchester, R. N. , McLaughlin, M. A. , Lyne, A. G. , Ferdman, R. D. , Burgay, M. , Lorimer, D. R. , Possenti, A. , D’Amico, N. , Sarkissian, J. M. , Hobbs, G. B. , Reynolds, J. E. , Freire, P. C. C. , Camilo, F., Tests of General Relativity from Timing the Double Pulsar, Science, 314, 97, (2006)

[7] Breton, R. P. , Kaspi, V. M. , Kramer, M. , McLaughlin, M. A. , Lyutikov, M. , Ransom, S. M. , Stairs, I. H. , Ferdman, R. D. , Camilo, F. , Possenti, A., Relativistic Spin Precession in the Double Pulsar, Science, 321, 104, (2008)

[8] Weisberg, J. M. and Taylor, J. H., Observations of post-Newtonian timing effects in the binary pulsar PSR 1913+16, Phys. Rev. Lett., 52, 1348, (1984)

[9] A. Abramovici , W. E. Althouse , R. W. P. Drever, Y. Gursel, S. Kawamura , F. J. Raab , D. Shoemaker, L. Sievers, R. E. Spero, K. S. Thorne, R. E. Vogt, R. Weiss , S. E. Whitcomb , M. E. Zucker, LIGO: The Laser Interferometer Gravitational-Wave Observatory, Science, 256, 325, (1992)

[10] Barish, B. C., The Laser Interferometer Gravitational-Wave Observatory LIGO, Adv. Sp. Res., 25, $1165,(2000)$

[11] Danzmann, K., LISA Mission Overview, Adv. Sp. Res., 25, 1129, (2000)

[12] R. W. Hellings and G. S. Downs, Upper Limits on the Isotropic Gravitational Radiation Background from Pulsar Timing Analysis, ApJ, 265, 39, (1983)

[13] Jenet, F. A., Hobbs, G. B., Lee, K. J., Manchester, R. N., Detecting the Stochastic Gravitational Wave Background Using Pulsar Timing, ApJ, 625, 123, (2005)

[14] van Haasteren, R., Levin, Y., McDonald, P., Lu, T., On measuring the gravitational-wave background using Pulsar Timing Arrays, MNRAS, 395, 1005, (2009)

[15] Kramer, M., Backer, D. C., Cordes, J. M., Lazio, T. J. W., Stappers, B. W., Johnston, S., Strong-field tests of gravity using pulsars and black holes, New A Rev., 48, 993, (2004)

[16] Hobbs, G. B. , Bailes, M. , Bhat, N. D. R. , Burke-Spolaor, S. , Champion, D. J. , Coles, W. , Hotan, A. , Jenet, F. , Kedziora-Chudczer, L., Khoo, J. , Lee, K. J. , Lommen, A. , Manchester, R. N. , Reynolds, J. , Sarkissian, J. , van Straten, W. , To, S. , Verbiest, J. P. W. , Yardley, D. , You, X. P., Gravitational-Wave Detection Using Pulsars: Status of the Parkes Pulsar Timing Array Project, PASA, 26, 103, (2009)

[17] Ferdman, R. D. , van Haasteren, R. , Bassa, C. G. , Burgay, M. , Cognard, I. , Corongiu, A. , D’ Amico, N. , Desvignes, G. , Hessels, J. W. T. , Janssen, G. H. , Jessner, A. , Jordan, C. , Karuppusamy, R. , Keane, E. F. , Kramer, M. , Lazaridis, K. , Levin, Y. , Lyne, A. G. , Pilia, M. , Possenti, A. , Purver, M. , Stappers, B. , Sanidas, S., Smits, R. , Theureau, G., The European Pulsar Timing Array: current efforts and a LEAP toward the future, Classical and Quantum Gravity, 27, 084014, (2010) 
[18] Jenet, F. , Finn, L. S. , Lazio, J., Lommen, A., McLaughlin, M. , Stairs, I. , Stinebring, D. , Verbiest, J. , Archibald, A. , Arzoumanian, Z. , Backer, D. , Cordes, J. , Demorest, P. , Ferdman, R. , Freire, P. , Gonzalez, M. , Kaspi, V. , Kondratiev, V. , Lorimer, D. , Lynch, R. , Nice, D. , Ransom, S. , Shannon, R., Siemens, X., The North American Nanohertz Observatory for Gravitational Waves, ArXiv e-prints, (2009), 0909.1058

[19] Hobbs, G. , Archibald, A. , Arzoumanian, Z. , Backer, D. , Bailes, M. , Bhat, N. D. R. , Burgay, M. , Burke-Spolaor, S. , Champion, D. , Cognard, I. , Coles, W. , Cordes, J. , Demorest, P. , Desvignes, G. , Ferdman, R. D. , Finn, L. , Freire, P. , Gonzalez, M. , Hessels, J. , Hotan, A. , Janssen, G. , Jenet, F. , Jessner, A. , Jordan, C. , Kaspi, V. , Kramer, M. , Kondratiev, V. , Lazio, J. , Lazaridis, K. , Lee, K. J. , Levin, Y. , Lommen, A. , Lorimer, D. , Lynch, R. , Lyne, A. , Manchester, R. , McLaughlin, M. , Nice, D. , Oslowski, S. , Pilia, M. , Possenti, A., Purver, M. , Ransom, S. , Reynolds, J. , Sanidas, S. , Sarkissian, J., Sesana, A., Shannon, R., Siemens, X. , Stairs, I. , Stappers, B. , Stinebring, D. , Theureau, G. , van Haasteren, R., van Straten, W. , Verbiest, J. P. W. , Yardley, D. R. B. , You, X. P., The International Pulsar Timing Array project: using pulsars as a gravitational wave detector, Classical and Quantum Gravity, 27, 084013, (2010)

[20] Stappers, B. and Kramer, M., Searching for Gravitational Waves with the EPTA and LEAP, in proceedings of American Astronomical Society Meeting Abstracts, 43, 124.04, (2011)

[21] Jenet, F. A., Hobbs, G. B., van Straten, W, Manchester, R. N., Bailes, M., Verbiest, J. P. W., Edwards, R. T., Hotan, A. W., Sarkissian, J. M., Ord, S. M., Upper Bounds on the Low-Frequency Stochastic Gravitational Wave Background from Pulsar Timing Observations: Current Limits and Future Prospects, ApJ, 653, 1571, (2006)

[22] Demorest, P. B. , Ferdman, R. D. , Gonzalez, M. E. , Nice, D. , Ransom, S. , Stairs, I. H. , Arzoumanian, Z. , Brazier, A. , Burke-Spolaor, S. , Chamberlin, S. J. , Cordes, J. M. , Ellis, J. , Finn, L. S. , Freire, P. , Giampanis, S. , Jenet, F. , Kaspi, V. M. , Lazio, J. , Lommen, A. N. , McLaughlin, M. , Palliyaguru, N. , Perrodin, D. , Shannon, R. M. , Siemens, X. , Stinebring, D. , Swiggum, J. , Zhu, W. W., Limits on the Stochastic Gravitational Wave Background from the North American Nanohertz Observatory for Gravitational Waves, ArXiv e-prints, (2012), 1201.6641

[23] van Haasteren, R. , Levin, Y., Janssen, G. H. , Lazaridis, K. , Kramer, M. , Stappers, B. W. , Desvignes, G. , Purver, M. B., Lyne, A. G. , Ferdman, R. D., Jessner, A. , Cognard, I. , Theureau, G. , D’Amico, N. , Possenti, A., Burgay, M. , Corongiu, A., Hessels, J. W. T. , Smits, R. , Verbiest, J. P. W., Placing limits on the stochastic gravitational-wave background using European Pulsar Timing Array data, MNRAS, 414, 3117, (2011)

[24] Sanidas, S. A., Battye, R. A., Stappers, B. W., Constraints on cosmic string tension imposed by the limit on the stochastic gravitational wave background from the European Pulsar Timing Array, Phys. Rev. D, 85, 122003, (2012)

[25] Freire, P. C. C., Wex, N., Esposito-Farèse, G., Verbiest, J. P. W., Bailes, M., Jacoby, B. A., Kramer, M., Stairs, I. H., Antoniadis, J., Janssen, G. H. , The relativistic pulsar-white dwarf binary PSR J1738+0333 - II. The most stringent test of scalar-tensor gravity, MNRAS, 423, 3328, (2012)

[26] Lazaridis, K., Verbiest, J. P. W., Tauris, T. M., Stappers, B. W., Kramer, M., Wex, N., Jessner, A., Cognard, I., Desvignes, G., Janssen, G. H., Purver, M. B., Theureau, G., Bassa, C. G., Smits, R., Evidence for gravitational quadrupole moment variations in the companion of PSR J2051-0827, MNRAS, 414, 3134, (2011) 